\title{
Article \\ Delaunay Mesh Construction and Simplification with Feature Preserving Based on Minimal Volume Destruction
}

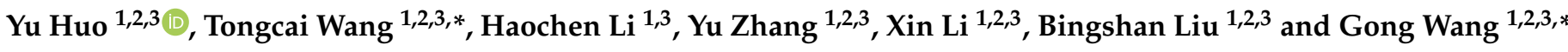 \\ 1 Technology and Engineering Center for Space Utilization, Chinese Academy of Sciences, \\ No. 9 Dengzhuang South Road, Beijing 100094, China; huoyu16@csu.ac.cn (Y.H.); lihaochen@csu.ac.cn (H.L.); \\ zhangyupaul0228@gmail.com (Y.Z.); lixin@csu.ac.cn (X.L.); liubingshan@csu.ac.cn (B.L.) \\ 2 University of Chinese Academy of Sciences, Beijing 100049, China \\ 3 CAS Key Laboratory of Space Manufacturing Technology, Beijing 100094, China \\ * Correspondence: wangtongcai@csu.ac.cn (T.W.); wanggong@csu.ac.cn (G.W.)
}

check for updates

Citation: Huo, Y.; Wang, T.; Li, H.; Zhang, Y.; Li, X.; Liu, B.; Wang, G. Delaunay Mesh Construction and Simplification with Feature Preserving Based on Minimal Volume Destruction. Appl. Sci. 2022, 12, 1831. https://doi.org/10.3390/app12041831

Academic Editor: Giancarlo Mauri

Received: 29 December 2021

Accepted: 8 February 2022

Published: 10 February 2022

Publisher's Note: MDPI stays neutral with regard to jurisdictional claims in published maps and institutional affiliations.

Copyright: (c) 2022 by the authors. Licensee MDPI, Basel, Switzerland. This article is an open access article distributed under the terms and conditions of the Creative Commons Attribution (CC BY) license (https:// creativecommons.org/licenses/by/ $4.0 /)$.

\begin{abstract}
Triangular meshes play critical roles in many applications, such as numerical simulation and additive manufacturing. However, the triangular meshes transformed from computer-aided design models using common algorithms may have many undesirable narrow triangles, which tends to affect the downstream applications. In this paper, we proposed two algorithms for Delaunay mesh construction and simplification to improve the quality of the triangular meshes. Two improved mesh operations of inserting vertices and collapsing vertices based on the principle of minimum volume destruction were designed. The improved vertex inserting operation is able to modify the local mesh so that it will conform to the local Delaunay property. The improved vertex collapsing operation can realize the simplification of the original mesh while maintaining the local Delaunay property. The results of visualized rendering and thermal diffusion simulations verified the improvement of the proposed algorithms in the aspects of the quantity and quality of the meshes.
\end{abstract}

Keywords: Delaunay mesh; construction; simplification; geodesic distance

\section{Introduction}

Computer-aided design (CAD) models have played crucial roles in technical fields, such as numerical simulation, additive manufacturing and visualization. Accordingly, the triangular mesh, generated from CAD models, has become the dominant type of functional element due to its advantages of efficiency, simplicity and flexibility. However, triangular meshes, which are always transformed directly from a CAD model, usually have the defects of excessively small or large angles, and redundant or irregular vertices. These defects tend to reduce the accuracy of the numerical simulation based on triangular meshes. Thus, it is necessary to establish effective remeshing algorithms to optimize the generated triangular meshes, which is important for the downstream applications.

Alliez et al. [1] summarized many theories and applications of remeshing algorithms, which can be divided into the quantity-dominant simplification algorithms and qualitydominant optimization algorithms. In the aspects of simplification algorithms, Hoppe et al. [2] presented a serious of basic mesh operations for mesh simplification. Garland and Heckbert [3] also proposed a kind of quadratic error metrics (QEM) simplification method, which is also regarded as the general model simplification algorithm. As for the optimization algorithms, Botsch and Konnelt [4] first introduced the Laplace-Beltrami (LB) operator to improve the quality of the irregular triangular meshes. Based on the LB operator, they were able to construct area-equalizing triangulations with vertex smoothing operation and optimize the original mesh, which was regarded as the basic optimization algorithms. Furthermore, Wang et al. [5] transformed the original judgment condition for edges into the angles, enabling the simplification after the mesh optimization. Wang et al. [6] have realized the constraint of the shape of the triangle by varying the size of bubble based 
on the dynamics simulation method. Khan et al. [7] also tried to plan a more reasonable distribution of the number of vertex-to-vertex connections and optimize the meshes by constraining the range of angles. Most of these algorithms were based on the method of area-equalizing triangulation, which were used to achieve optimization and simplification of meshes in forms of regular triangles. Although the regular triangles can improve the computational accuracy of simulation results, the overemphasis on the evenly constructed triangular elements may limit the reduction of data storage and cause destruction to the geometric feature information of the original model. It is considered that the accuracy of numerical simulations can be improved when the cotangent formula $\cot \alpha+\cot \beta$ of the discrete LB operators has non-negative weight. According to this theory, a geodesic distance based on the discrete LB operator was used to simulate the thermal diffusion by Crane et al. [8]. Moreover, Bobenko and Springborn [9] proved that the Delaunay triangulation could always satisfy the non-negative weight for discrete LB operators, which was defined as the local property of the Delaunay triangulation. Correspondingly, the global property of the Delaunay triangulation was defined as the maximization of all the minimum angles in the triangulation, which can be used to indirectly constrain the shape of the triangle and avoid narrow triangles [10]. It is obvious that the Delaunay triangulation has the advantages in protecting the geometric features and simplifying the original meshes. However, it is difficult to achieve the Delaunay triangulation on the Riemannian manifold, considering that the geodesic paths are difficult to be directly represented with existing data structures. To solve this problem, Dyer et al. [11] defined a special concept of Delaunay mesh that, as long as the sampling criterion acquired a relatively lower bound among the distances of vertices in the Voronoi diagram, the Delaunay mesh can be obtained. Both Dyer et al. [12] and Liu et al. [13] proposed their adaptive sampling criterions to convert an arbitrary mesh into the Delaunay mesh by splitting non-Delaunay edges. Their methods tended to increase the number of vertices while the original mesh was converted to the Delaunay mesh. Therefore, the computational complexity including space complexity of data storage and time complexity of model processing will be inevitably increased, which may limit the application and development of Delaunay triangulation, especially for the case of original meshes with narrow triangles. Although Yi et al. [14] proved a hybrid mechanism operation of edge splitting and collapsing based on differential equations, which were able to reduce the complexity of the computational processing, the basic principle of algorithms has not been optimized.

In this paper, we propose two algorithms for the construction and simplification of Delaunay meshes. The Delaunay construction algorithm can transform arbitrary triangular meshes into the Delaunay meshes with the same topological type. Because the positions of the inserted vertices are calculated according to the geometric information of the mesh, and the identification criteria of the narrow triangles are designed to terminate the algorithm loop, both the space complexity and the time complexity of the Delaunay mesh construction algorithm can be optimized. In addition, a simplification algorithm is designed to simplify the inserted vertices during the construction process, and then it will decrease the data storage of the constructed meshes. The hybrid algorithms can preserve the geometric features of the original CAD model with the principle of minimal volume destruction. The experiments show that the proposed algorithms were effective for optimizing the non-Delaunay meshes, and especially for the improvement of the quality and quantity of the Delaunay meshes.

\section{Related Work}

\subsection{Definition of Delaunay Triangulation}

The Delaunay triangulation has similar effects in the numerical simulation with the regular triangulation. It is defined as its duality with the Voronoi diagram, which is shown as the dashed lines in Figure 1. The region of the Voronoi diagram $R_{i}$ is divided according to the nearest neighbor principle, where point is associated with its nearest neighbor region. $R_{i}$ can be expressed as the following: 


$$
R_{i}=\left\{p \mid d\left(p, v_{i}\right) \leq d\left(p, v_{j}\right), j=0,1,2, \ldots, n, i \neq j\right\}
$$

where $p$ is any point in the defined space and $d\left(p, v_{i}\right)$ is the distance between $p$ and $v_{i}$. In particular, as the dual graph with the Voronoi diagram, the Delaunay triangulation mesh has the local property, which is also named as the empty geodesic circumcircle property. This means that, for a triangle $t$ consisting of vertices $v_{0}, v_{1}, v_{2}$ in the Delaunay triangulation mesh $M$, no other vertices that are not the vertex of the triangle in the circumcircle of $t$ exist. The local property implies that the commonly used cotangent Laplace-Beltrami operator has non-negative weights [13], which enables the Delaunay triangulation to have the similar performance as the area-equalizing triangulation in the numerical simulation.

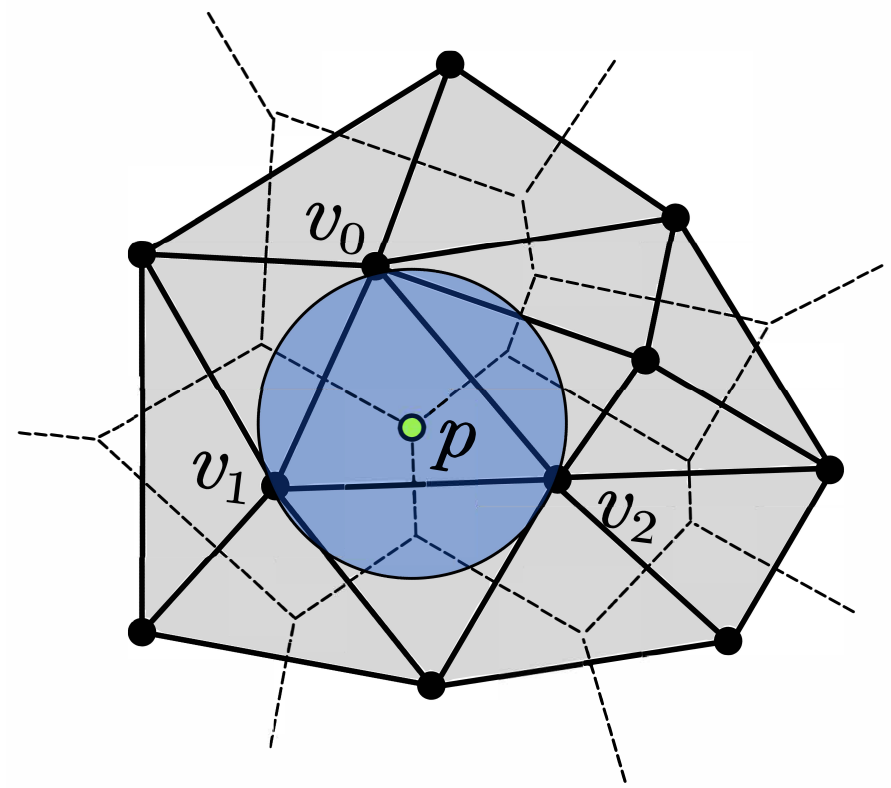

Figure 1. The illustration of the local Delaunay property.

\subsection{Intrinsic Delaunay Triangulation}

Rivin et al. [15] first defined the intrinsic Delaunay Triangulation on the manifold surface. Then, Leibon et al. [16] proved that a unique Delaunay triangulation existed on each closed Riemannian manifold, but it needs a large number of vertices to construct Delaunay triangulation this way. Since the geodesic paths, also known as the triangular edges of iDT, do not uniquely exist on the Riemannian manifold, it is necessary to confine the domain to a sufficiently small area by vertices. Chen et al. [17] presented a twodimensional Delaunay mesh smoother based on the optimal Delaunay triangulation (ODT), and they also tried to extend the ODT to three-dimensional conditions. However, on the Riemannian manifold, it is not easy to directly transform arbitrary triangle mesh into the Delaunay mesh. Because the geodesic paths are difficult to directly represent by existing data structures, one indirect method was proposed by using a Geometric Voronoi Diagram (GVD) [18,19], namely the dual of the Delaunay triangle, as an intermediate element. Liu et al. [20] proposed a Centroidal Voronoi Tessellation (CVT) approach that was proved to be able to construct high quality meshes. The classical Lloyd's iterations for CVT computing are linearly convergent and inefficient. Then, some algorithms are built to improve the efficiency of this kind of indirect construction algorithm by increasing the speed of solving. Liu et al. [21] proposed a quasi-Newton method to accelerate the speed of convergence. Wang et al. [22] summarized and compared the aforementioned methods and the limited-memory BFGS (L-BFGS) method in detail. They also contrived a way to efficiently compute the Riemannian center on the basis of the discrete exponential map. Subsequently, Liu et al. [23] presented a novel method relying on manifold differential evolution (MDE), and obtained the globally optimal geodesic on triangle meshes. 


\section{Method}

In this section, we first give the necessary symbol definitions and several standard local mesh operations. Then, two important improved mesh operations and the algorithm based on them are designed.

\subsection{Symbol Definition and Standard Mesh Operations}

Given an input mesh $M=(V, E, F)$ is a closed 2-manifold triangle mesh, where $V, E, T$ are the sets of vertex, edge, and triangular element, respectively, for an edge $e_{i j}$, vertices $\left(v_{i}, v_{j}\right)$ are the endpoints of it. The definition of the 1-ring neighborhood $\Omega\left(v_{i}\right)$ of $v_{i} \in V$ is shown in Figure 2a:

- For vertex $v$, if the $v \in \Omega\left(v_{i}\right)$, the set $\{v\}$ is all of the vertices adjacent to $v_{i}$, as shown in red vertices in Figure 2a.

- For edge $e_{i j}$, if $e_{i j} \in \Omega\left(v_{i}\right)$, the set $\left\{e_{i j} \mid 1 \leq i, j \leq n\right\}$ is all of the edges whose endpoints are the pair of vertices $\left(v_{i}, v_{j}\right)$.

- For triangle $t_{i j k}$, if $t_{i j k} \in \Omega\left(v_{i}\right)$, the set $\left\{t_{i j k} \mid 1 \leq i, j, k \leq n\right\}$ is all of the triangles incident to vertex $v_{i}$.

- In particular, for edge $e_{j k}$, if $e_{j k} \in t_{i j k}$ and $t_{i j k} \in \Omega\left(v_{i}\right)$, the set $D\left(v_{i}\right)$ could denote all $e_{j k}$ which satisfy the condition, as indicated by the blue lines in Figure 2a.

Figure $2 \mathrm{~b}$ shows a series of standard local mesh operations. These operations were originally designed for the mesh simplification algorithm [2]. The effect on the local Delaunay properties of the mesh is not considered when the operations are executed. It is necessary to improve them to make these operations more suitable for Delaunay mesh construction and simplification algorithms.

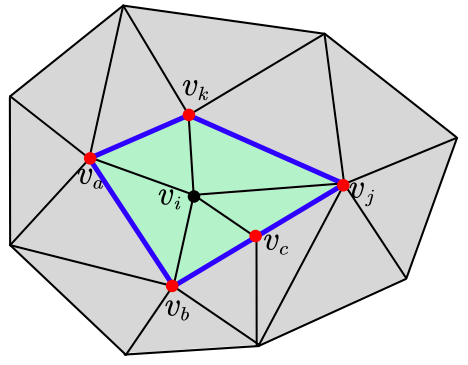

(a) 1ring neighborhood

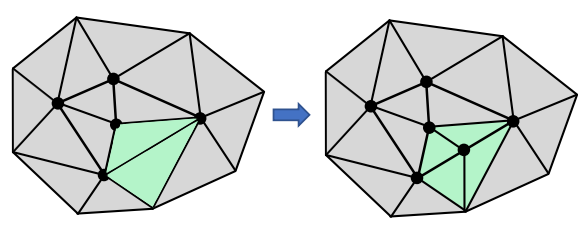

(b1) Edge splitting

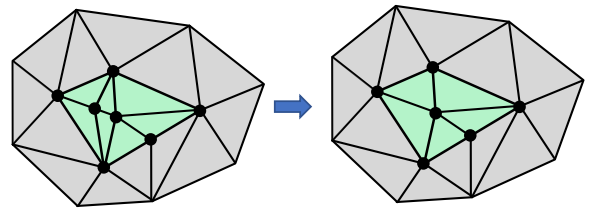

(b2) Edge collapsing

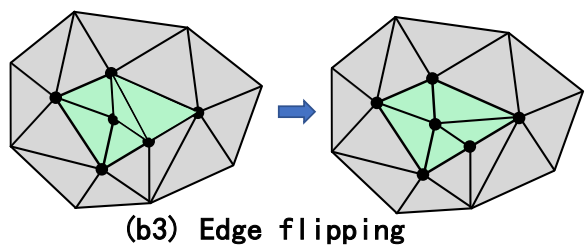

Figure 2. (a) Schematic of a 1-ring neighborhood $\Omega\left(v_{i}\right)$; (b) standard operations for the remeshing algorithm.

\subsection{The Delaunay Mesh Construction Algorithm}

The vertex inserting is considered as an improved version of the edge splitting. For a local non-Delaunay mesh $M$, it is possible to satisfy the local Delaunay property by choosing the appropriate position for inserting a new vertex. First, an assumption of a general situation and related inferences is given.

As shown in Figure 3, the local non-Delaunay mesh $M$ consists of four vertices $v_{i}, v_{g}, v_{j}$ and $v_{k}$. For a non-locally Delaunay edge $e_{i j}$, the sum of the two opposite angles $\angle v_{i} v_{k} v_{j}$ 
and $\angle v_{i} v_{g} v_{j}$ is greater than $\pi$. In this case, at least one of the two opposite angles of $e_{i j}$ exists as an obtuse angle, and $e_{i j}$ is the longest edge of this obtuse triangle $t_{i j k}$. Such that $e_{i j}$ is not the shortest edge in the set $E_{i}=\left\{e_{i j} \mid e_{i j} \in \Omega\left(v_{i}\right)\right\}$ because there must exist both $e_{i j}$ and $e_{i k}$ belonging to $\Omega\left(v_{i}\right)$. Thus, we assume that edge $e_{i a} \in E_{i}$ is the shortest edge of $E_{i}$. As shown in Figure 3, the same length on $e_{i j}$ is intercepted at point $v_{a}^{\prime}$, and $O$ is the midpoint of this length. Given any inserted vertex $s$ on the line between $O$ and $v_{a}^{\prime}$, the intersection of the gray area with $e_{i j}$ is the targeted range of $s$. Considering the relation of $l_{A v_{i}}=l_{s v_{i}}=l_{B v_{i}}$, the suitable location can be determined by the following conditions:

$$
\angle v_{i} v_{k} s+\angle v_{i} v_{g} s \leq \angle v_{i} A s+\angle v_{i} B s=\frac{2 \pi-\angle v_{k} v_{i} v_{g}}{2}<\pi,
$$

Similarly, we can also obtain a vertex $s^{\prime}$ for the case of $v_{j}$. If the regions of $s$ and $s^{\prime}$ are overlapped, the inserted vertex is chosen as the midpoint in the regions. However, if these regions are not overlapped, the above process can be repeated and the convergence of it are acquired due to the monotonically of $s u m_{1}=\angle v_{i} v_{k} s+\angle v_{i} v_{g} s$ and $s u m_{2}=\angle s v_{k} v_{j}+\angle s v_{g} v_{j}$. As the region where $s$ is taken gradually moves away from $v_{i}$ to $v_{j}$,sum $m_{1}$ monotonically increases and sum 2 monotonically decreases. Both of them are less than $\pi$, which means $e_{i s}$ and $e_{s j}$ can fulfill the local Delaunay property. The improved vertex inserting operation can choose the suitable location for the newly inserted vertex $s$, and then the local Delaunay property can be achieved. After the improved vertex inserting operation, the Delaunay property of the region consisting of four vertices $v_{i}, v_{g}, v_{j}$ and $v_{k}$ is satisfied. These new angles $\angle v_{i} s v_{k}, \angle v_{i} s v_{g}$ and $\angle v_{j} s v_{g}$ destroy the local Delaunay property of the surrounding region, so the repeated vertex inserting is acceptable. However, in the case of narrow triangle appearance, it may repeat the vertex inserting operation endlessly. Therefore, a control parameter $\delta$ should be designed to detect narrow triangles and avoid this kind of useless repetitive operation.

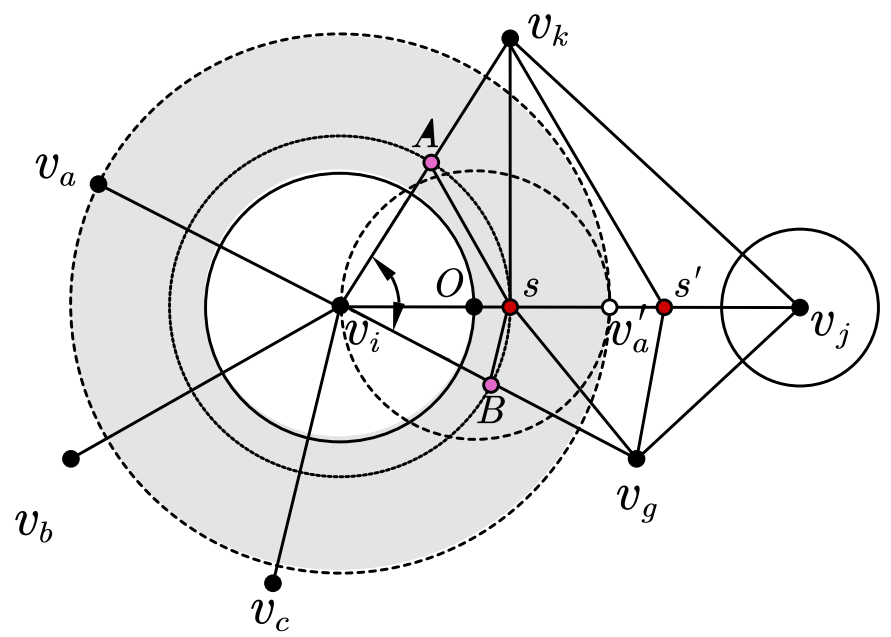

Figure 3. Choosing the position of newly insert vertex. For the original vertex $v_{i}$, the newly inserted vertex $s$ on the line segment between $O$ and $v_{a}^{\prime}$. Similarly, for the $v_{j}$, an inserted vertex $s^{\prime}$ is been obtained.

For the original mesh $M, l_{\min }$ is the length of the shortest edge. $\theta_{\min }$ is the smallest angle. $h$ is the distance between vertex $v_{k}$ and bottom edge $e_{i j}$ of triangle $t_{i j k}$. $d$ is the distance between vertex $v_{k}$ and inserted vertex $v_{s}$. For a Delaunay mesh $D M$ complied with the local Delaunay property, all the edges of $D M$ satisfy the following conditions:

- Conditions 1: As shown in Figure 4a, for any triangle $t_{i j k}$ in $M$, this state is always satisfied:

$$
d \geq h=l \cdot \sin (\theta) \geq l_{\min } \cdot \sin \left(\theta_{\min }\right),
$$


- Conditions 2: For original edge $e$ in $M$, the condition is also found that:

$$
\min \left\{l_{e} \mid e \in \Omega\left(v_{i}\right)\right\} \geq l_{\text {min }} \cdot \sin \left(\theta_{\text {min }}\right),
$$

- Conditions 3: As shown in Figure 4b, the distance of the newly inserted vertex from any vertex also satisfies that:

$$
l_{\text {is }} \geq l_{\text {ig }} \cdot \sin \left(\theta_{\text {min }}\right) \geq l_{\text {min }} \cdot \sin \left(\theta_{\text {min }}\right),
$$

Therefore, if a new edge $e_{s g}$ is shown in Figure $4 \mathrm{~b}$, whose length is less than $l_{\text {min }}$. $\sin \left(\theta_{0}\right)$, the triangle $t_{i j g}$ could be considered as a narrow triangle. In the practical application, the $l_{\text {min }}$ and $\sin \left(\theta_{\text {min }}\right)$ do not exist in the same triangle. Thus, a control factor $\delta$ could be set as $\delta=k \cdot l_{\min } \cdot \sin \left(\theta_{0}\right), k \in Z^{+}$to replace $l_{\min } \cdot \sin \left(\theta_{0}\right)$. In other words, if the length of a new edge $e_{s g}$ is less than $\delta$, the triangle will be considered as a narrow triangle which is collapsible in the algorithm and shown in Figure 4c. This condition is considered as the case of volume destruction to the original mesh because it makes the final narrow triangle compressed into a single edge.

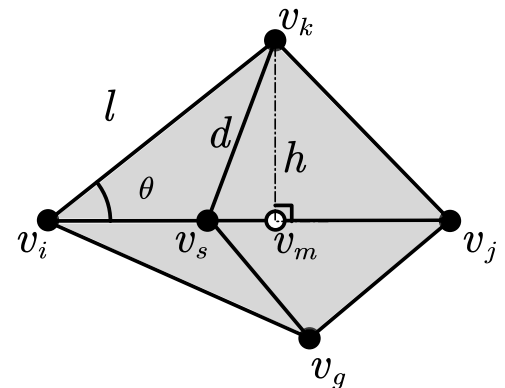

(a) Case 1

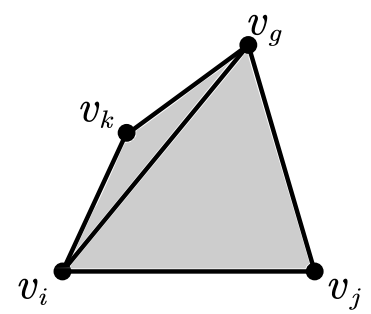

(c) Face collapsing

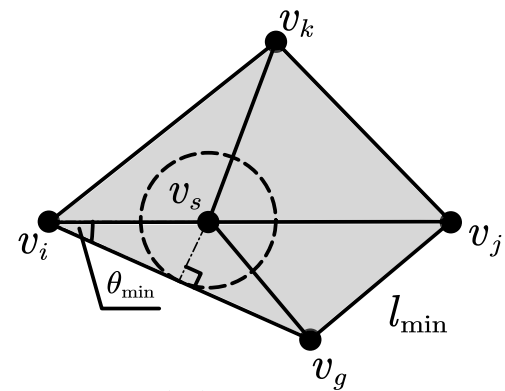

(b) Case 3

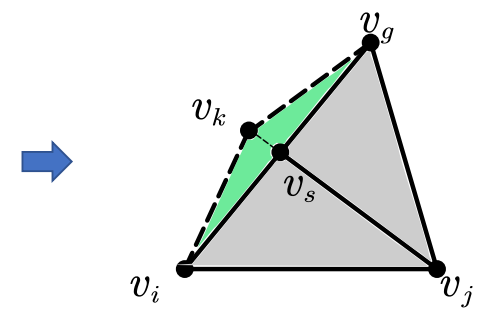

Figure 4. (a) Schematic diagram of Case 1; (b) schematic diagram of Case 3; (c) face collapsing.

A Delaunay mesh construction algorithm is designed and the pseudo code is shown in Algorithm 1. We try to implement the stack using the priority queue approach. Firstly, the control factor $\delta$ is initialized. Then, we place all non-local Delaunay edges in a priority queue $Q$ keyed on the sum of the diagonals corresponding to edge $e$ with the largest sum at the top. The algorithm iterative inserts a newly vertex $v_{s}$ to split the NLD edge from the queue, and updates the information about all elements in $\Omega\left(v_{S}\right)$ such as connectivity and normal vectors. Finally, the edges in the $D\left(v_{s}\right)$ should be determined if them are the NLD edges and all of the new NLD edges will be pushed in the priority queue $Q$. The algorithm will repeat this procedure until the priority queue $Q$ is empty. 


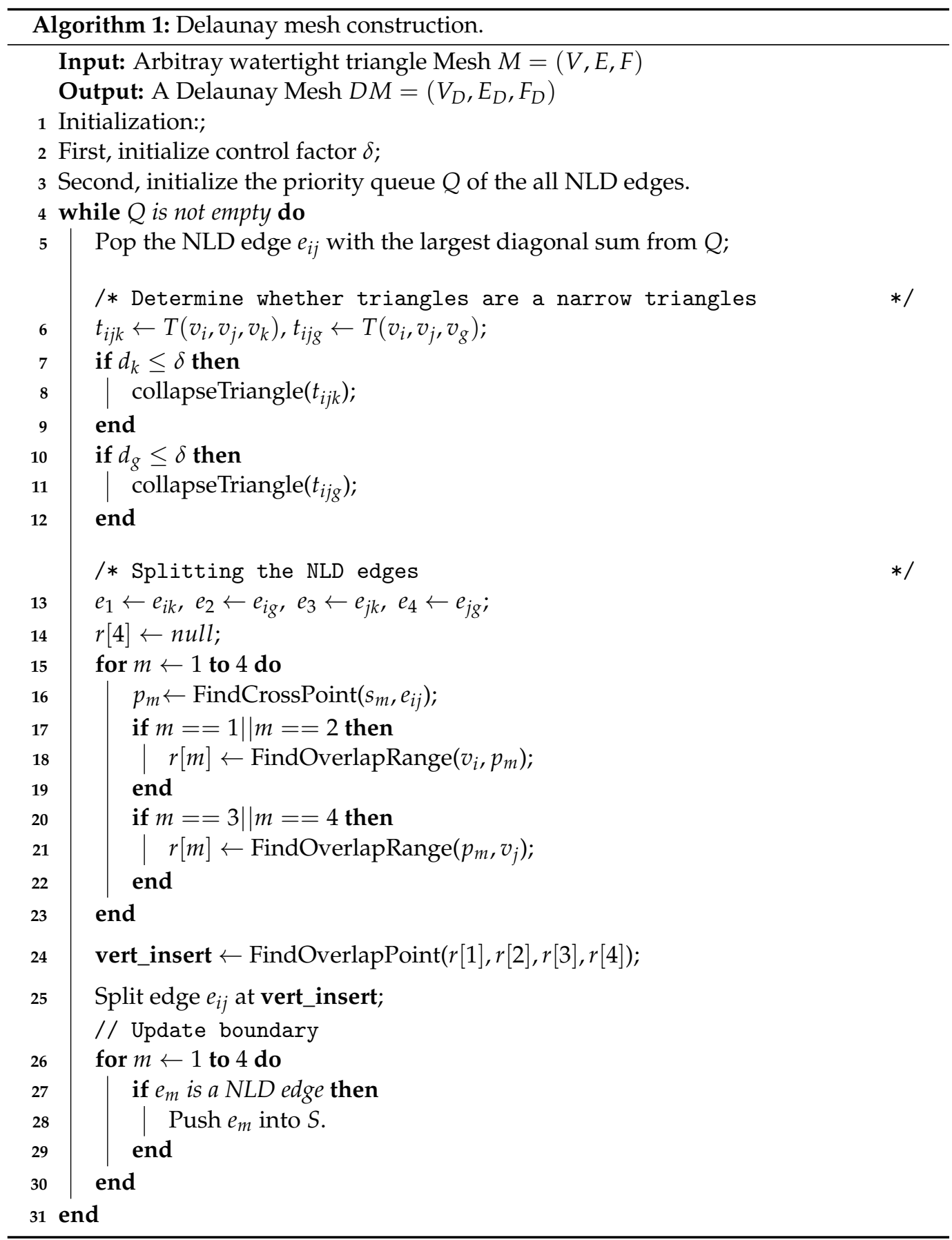

\subsection{The Delaunay Mesh Simplification Algorithm}

The complexity of a mesh will increase because of the newly inserted vertices according to the vertex inserting operation. An improved vertex collapsing operation is designed to conquer this drawback. The requirement for the improved vertex collapsing operation is that the local Delaunay property should be preserved in the non-connected domain. We assume that the original mesh is a Delaunay mesh, and the all edges in the set of hollow edges satisfied the local Delaunay property. As shown in Figure 5a, a hollow would be created if a vertex was deleted, and an improved vertex collapsing operation is designed to fill the hollow created by the vertex deletion, whose output is still a Delaunay mesh. 

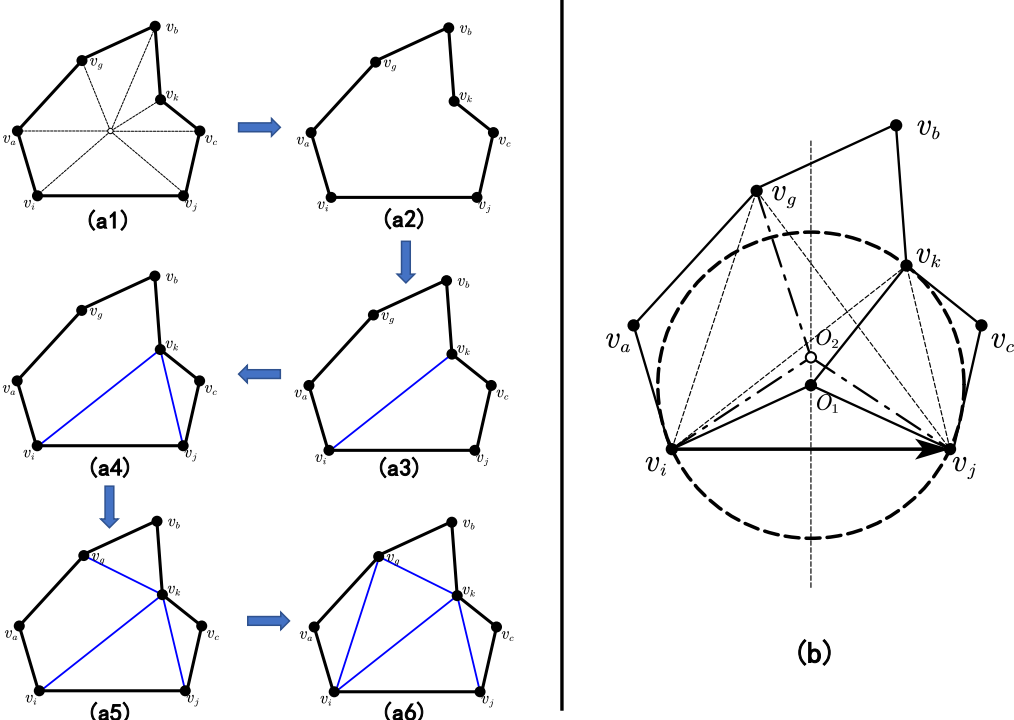

(b)

Figure 5. (a) Schematic diagram of hole filling; (b) principle of vertex collapsing.

The principle of the improved operation is shown in Figure $5 \mathrm{~b}$. The edge $e_{i j}$ is in the set of hollow-edges, and the set of vertices $V$ is all the vertices that are located in the hollow-edges. We assume that $e_{i j}$ is a directed edge with the certain direction from vertex $v_{i}$ to vertex $v_{j}$. From the principle of the empty geodesic circumcircle property, for any triangle containing edge $e_{i j}$, there are no other vertices in its circumcircle. Thus, the key of vertices collapsing operation is to find the minimum distance between the circumcenter $O$ of a triangle $t$ and the edge $e_{i j}$. The specific situation is represented as shown in Figure $5 \mathrm{~b}$, where $O_{1}$ is the circumcenter of $t_{i j k}$, and the distance from $O_{1}$ to $e_{i j}$ is the shortest; then, the triangle $t_{i j k}$ will satisfy the empty geodesic circumcircle property. Setting the direction of an edge and finding a vertex to the left of $e_{i j}$ can avoid errors in the results because of the concave vertex just like $v_{k}$. After all the edges in the set of hollow-edges rebuild the required vertices, the hollow would be patched. The local-Delaunay property of the interior edges, which are inserted after hollow-patching, could be preserved as well.

It is obvious that the improved vertex collapsing operation could be able to simplify a Delaunay mesh. Even if there are NLD edges in the original mesh, this operation can still work on the vertex $v_{i}$ as long as the edges $e_{j k} \in D\left(v_{i}\right)$ satisfy the local Delaunay property. Therefore, a simplification algorithm based on minimal volume destruction can be designed. The pseudo code is named as the pseudo-code Algorithm 2, where the iterative cost of $v_{i}$ is set as the volume of $\Omega\left(v_{i}\right)$ (see Figure 6). Firstly, we classify vertex $v_{i}$ as the non-collapsible and collapsible vertex according to the number of NLD edges in $D\left(v_{i}\right)$, respectively. If there are no NLD edges in $D\left(v_{i}\right)$, the vertex $v_{i}$ is classified as the collapsible vertex. In this cases, all edges $e_{j k} \in D\left(v_{i}\right)$ satisfy the local Delaunay property, and the improved vertex collapsing operation is worked on $v_{i}$, which will not destruct the local Delaunay property of all edges in $D\left(v_{i}\right)$. On the contrary, if there are NLD edges in $D\left(v_{i}\right)$, the improved vertex inserting operation would choose suitable locations on the NLD edge for newly inserted vertices. Then, a hybrid mechanism combined the improved vertex inserting operation and vertex collapsing operation will repeat until the number of vertices in the simplified mesh DM matches the user-defined value. 

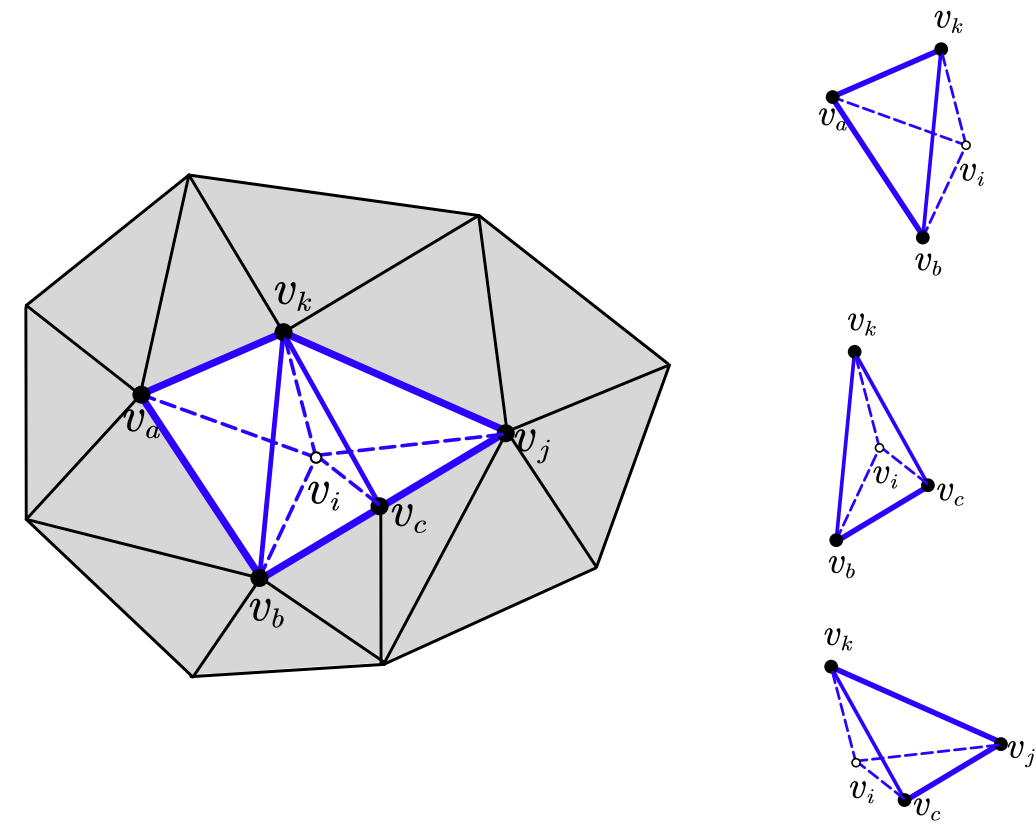

Figure 6. The expression of the sum of volume as an iterative constraint.

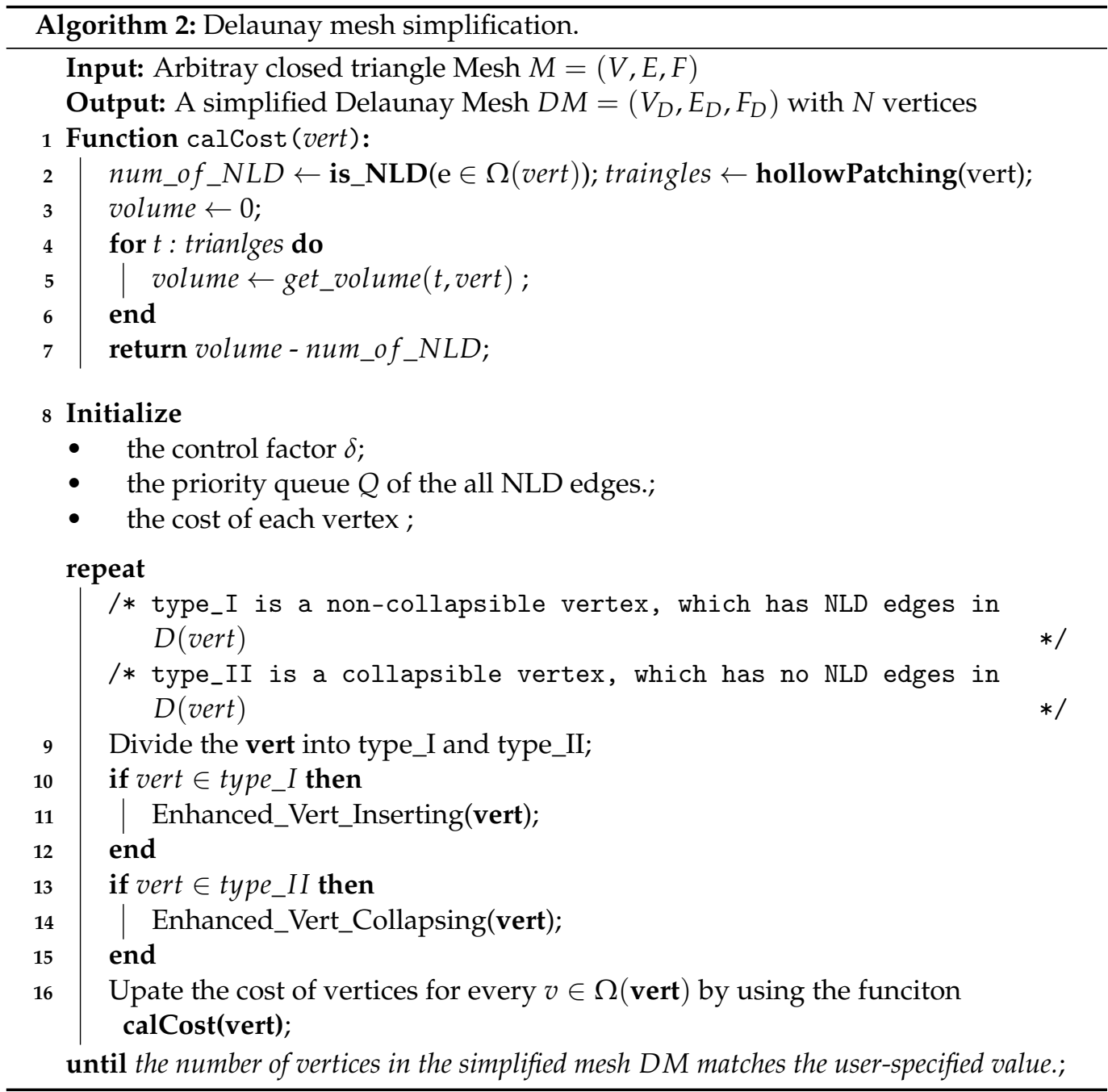




\section{Experimental and Discussion}

In this section, experimental results of the construction and simplification algorithm are demonstrated and discussed. All these results are conducted and obtained on a PC with an Intel Core i7-8750H CPU 2.20 GHz and 16.0 GB RAM, and all the codes are implemented in $\mathrm{C}++$.

\subsection{Evaluations of the Algorithm Effectiveness}

In this paper, the performance of the proposed algorithms is defined as the degree of geometric feature restoration of the original meshes and the accuracy of numerical simulation. Accordingly, two kinds of measures are proposed to evaluate the effectiveness of the algorithms. Firstly, for the degree of geometric feature restoration of the original meshes, the use of visual renderings allows for the visual and intuitive assessment. In addition, volume and surface area are the basic geometric information of a model. We set the geometric feature parameter $r$ as the ratio is a surface-area-to-volume to measure the geometric information of the triangular mesh. Volume, surface area, and their ratio $r$ can be calculated by the following equations, where $v_{i}, v_{j}, v_{k}$ are vertices on a triangle $t_{i j k}$ :

$$
\left\{\begin{array}{l}
\text { volume }=\sum \frac{1}{6}\left(v_{i} \times v_{j}\right) \cdot v_{k}, \\
\text { surface_area }=\sum \frac{1}{2}\left(v_{j}-v_{i}\right) \times\left(v_{k}-v_{i}\right), \\
r=\frac{\text { surface_area }}{\text { volume }},
\end{array}\right.
$$

Secondly, to demonstrate the effectiveness of numerical simulations, we used a geodesic distance based on the gradient calculation to simulate the thermal diffusion of isotropic simulation [8]. The accuracy of the computation of the scalar function determines the accuracy of the final numerical simulation, where the discrete Laplace operator is defined as a key factor, in the computation of the gradient of the scalar function. In particular, when calculating the gradient of thermal diffusion (see Figure 7), it can be specifically expressed as

$$
\Delta u_{i}=\frac{1}{2 A_{i}} \sum\left(\cot \alpha_{i j}+\cot \beta_{i j}\right)\left(u_{j}-u_{i}\right)
$$

Here, $u$ is a scalar function defined on the triangle mesh, and $A_{i}$ is one third of the area of all triangles incident on vertex $v_{i}$. The sum is calculated by adding up all neighboring vertices $v_{j} \in \Omega\left(v_{i}\right)$, where $\alpha_{i j}, \beta_{i j}$ are the angles opposing to the corresponding edge $e_{i j} \in \Omega\left(v_{i}\right)$.

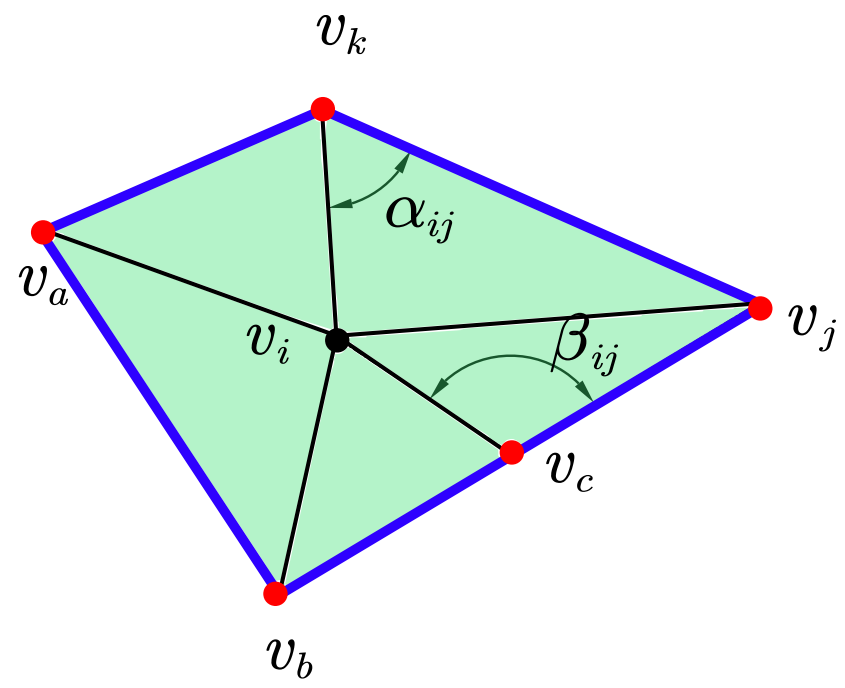

Figure 7. The cot formula for a scalar function on the mesh. 


\subsection{Performance of the Construction Algorithm}

Algorithm 1 is supposed to transform an arbitrary triangular mesh into a Delaunay mesh by inserting a finite number of vertices. For each NLD edge in the original mesh, the inserting operation may change the local Delaunay property of the edge. When there are narrow triangles connected to the NLD edge, it will result in more inserting vertices. As mentioned in Section 3.2, the control factor $\delta$ is defined to identify the narrow triangles and the improved face collapsing operation is used to handle this situation. Thus, for the construction algorithm of Delaunay mesh, $\delta$ is the most important parameter that determines the space complexity, represented by the number of vertices of the constructed Delaunay mesh. The theoretical lower bound of $\delta$ is $l_{\min } \cdot \sin \left(\theta_{\min }\right)$.

In this experiment, $\delta=2$. Average_length $\cdot \sin 15^{\circ}$ was selected as the suitable empirical parameter for the algorithms, which was able to achieve good performance for all the experimental meshes. In Figure 8, a triangle mesh "dinosaur" is used to demonstrate the effects of variable $\delta$. We take $l_{\min } \cdot \sin \left(\theta_{\min }\right)$ as the smallest value and $2 *$ Average_length $* \sin 15^{\circ}$ as the largest value, and select five data in that range to displace the relationship between the number of vertices and $\delta$. The rendering results of the smallest and largest values are shown. As shown in Figure 8a, the red curve depicts the relation between the control factor $\delta$ and the number of vertices of the constructed Delaunay mesh. With the increase of control factor $\delta$, the judgment criterion of the narrow triangles is amplified. Fewer vertices will be inserted because the face collapsing operation will be used before that. The geometric feature information of the triangle mesh is represented by the parameter $r$, which is the ratio of surface area to volume of the triangular mesh. As the control factor $\delta$ increases, the change of the parameter $r$ remains almost constant. This indicates that the variation of the control factor $\delta$ within a reasonable range has little effect on the geometric feature information of the Delaunay mesh. The constructed Delaunay meshes obtained by the two special control factors are rendered as shown in Figure $8 \mathbf{b}$. When the control factor is set as $\delta=l_{\min } \cdot \sin \left(\theta_{\min }\right)$, more vertices will be inserted because the narrow triangles generated from the face collapsing operation are too small. If the control factor is set as $\delta=2 *$ Average_length $* \sin 15^{\circ}$, the density of vertices in local areas can be effectively improved.

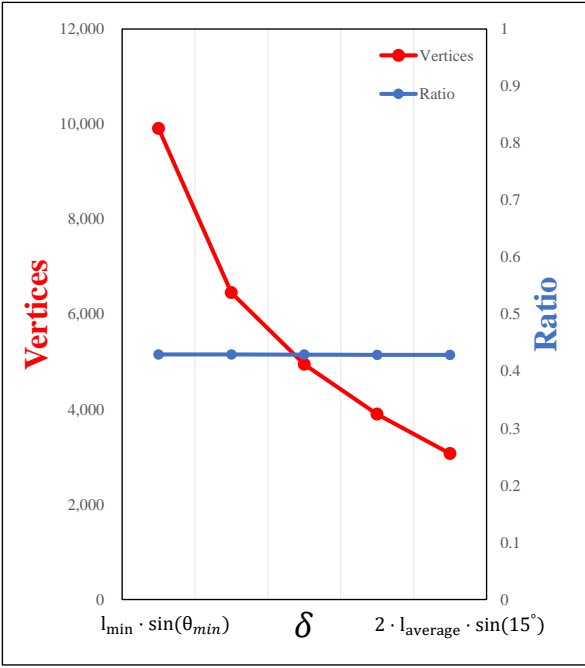

(a) Relationship of the parameters to $\delta$

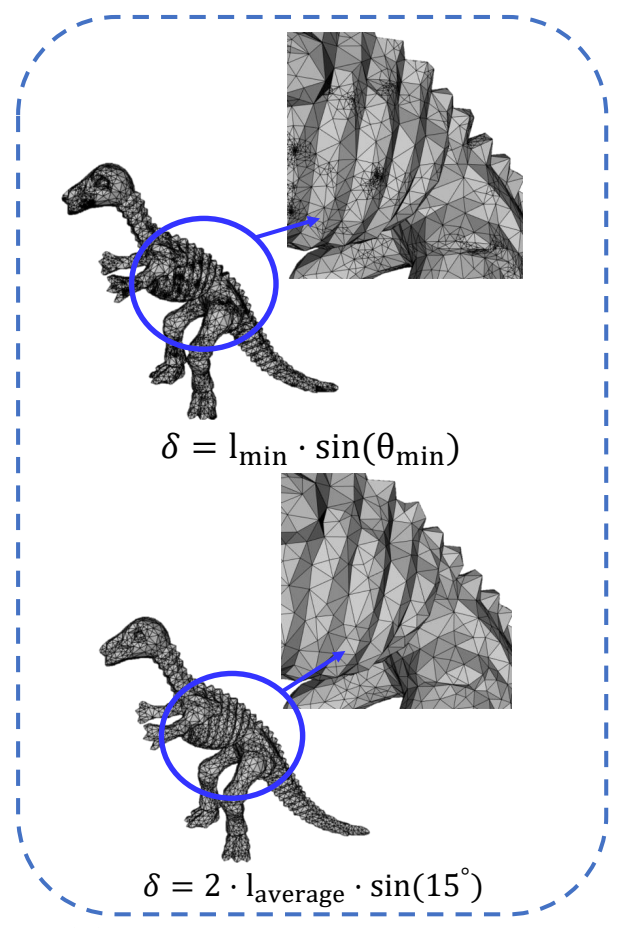

(b) Meshes with different $\delta$

Figure 8. The results of parameter $\delta$ selection with the model of "dinosaur". 
The mesh renderings demonstrate the preservation of the geometric feature information. The thermal diffusion renderings are performed to illustrate the calculation effectiveness in numerical simulations. In Figure 9a, the original mesh "refinement" has 510 vertices. Due to the presence of many NLD edges in the original mesh, the results of its thermal diffusion simulation have many errors. For example, in the region $\mathrm{A}$, the isotherms represented by the black line are not consistent with uniform diffusion. In the region $\mathrm{B}$, necessary data information is missing because of too few vertices. The above two conditions show that a non-Delaunay mesh has the lower accuracy in numerical simulation. Figure $9 \mathrm{~b}$ shows the constructed Delaunay mesh and the thermal diffusion rendering of it. After the processing of Algorithm 1, the Delaunay mesh with 688 vertices is obtained. It can be demonstrated that the constructed Delaunay mesh with 688 vertices can avoid the errors in the thermal diffusion simulation compared to results of the original mesh. In addition, we can find by the geometric feature parameter $r$ that the algorithm proposed in this paper has a better preservation of the geometric feature information of the mesh. In order to demonstrate the performance of the proposed method, the results of the classical area-equaling triangulation method [4] and the Delaunay mesh construction algorithm [13] are performed with the same model and shown in Figure 9c and Figure 9d, respectively. The selected area-equaling triangulation method is a basic algorithm for model meshing of finite element method (FEM), and Liu's method [13] is a typical representation for the construction of the Delaunay mesh. The number of vertices using their methods are 7003 and 7907, respectively. It is obvious that the construction algorithm proposed in this paper has better performance in the aspects of simulation accuracy with less vertex numbers.

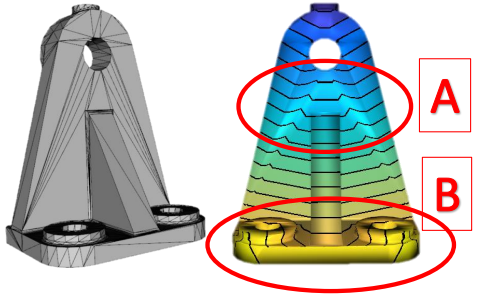

(a) Original mesh

$|V|=510, r=0.287$

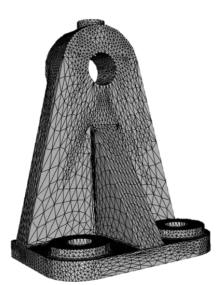

(c) Area-equalizing method $|V|=7003, r=0.287$

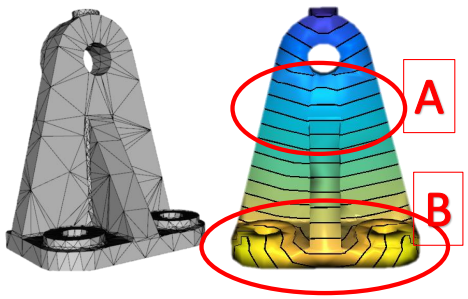

(b) Our method

$|V|=688, r=0.285$
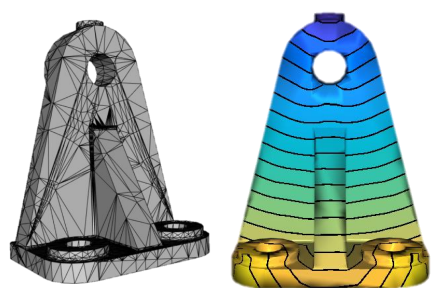

(d) Liu's method $|V|=7907, r=0.287$

Figure 9. The comparison of the construction algorithms with numerical simulation results, where regions $\mathrm{A}$ and $\mathrm{B}$ are significant changes.

\subsection{Performance of the Simplification Algorithm}

After the construction algorithm of Delaunay mesh via Algorithm 1, any triangle mesh can be transformed to the Delaunay mesh. However, the inserted vertices may lead to the increase of the mesh complexity and the data storage. Algorithm 2 provides a Delaunay mesh simplification algorithm that can maintain the local Delaunay property of the Delaunay mesh and transform the non-Delaunay mesh to the Delaunay mesh.

A model of "bumpy" is used to show the effect of the simplification algorithm on the preservation of the geometric information. The original mesh with 1250 vertices is the Delaunay mesh, and the improved vertex collapsing operation is used to simplify it. As shown in Figure 10, the un-simplified mesh and the simplified meshes with $80 \%$ vertices, $40 \%$ vertices, and $20 \%$ vertices are displayed, respectively. Both the visualization and the 
ratio $r$, surface-area-to-volume, demonstrate that there is better preservation of geometric information as the number of vertices of the simplified mesh is reduced.

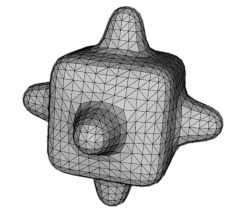

$|V|=1250, r=1.105$

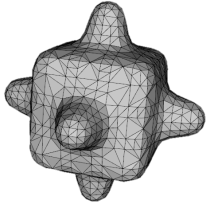

$|V|=1000, r=1.108$

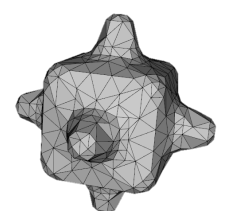

$|V|=500, r=1.114$

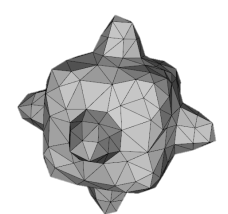

$|V|=250, r=1.105$

Figure 10. The simplification results for a Delaunay mesh using the model of "bumpy".

Similarly, the simplification algorithm also works for non-Delaunay mesh. The variation trends of the number of vertices and the number of NLD edges with the iteration steps are shown in Figure 11. The blue curve indicates the trend of vertices versus iteration steps, and the red curve represents the trend of NLD edges versus iteration steps. The input mesh of Algorithm 2 is a non-Delaunay mesh with 9397 vertices and 1082 NLD edges, which are illustrated as A. The processing of algorithm can be divided into two parts. According to the simplification algorithm described in Section 3.3, for a non-Delaunay mesh, it firstly classifies all vertices into two types. For all of the non-collapsible vertices, it can be found that the number of the mesh vertices increased with the number of iterations of the algorithm in region-I of Figure 11a. Subsequently, when all the vertices in the mesh are collapsible vertices, which is illustrated as $B$, the number of vertices start to decrease. As the iterative step proceeds, the Delaunay mesh based on minimal volume destruction can be obtained, which is illustrated as C. In the region-II of Figure 11a, the NLD edges disappear, and the simplification algorithm is executed repeatedly until the user defined value is satisfied.

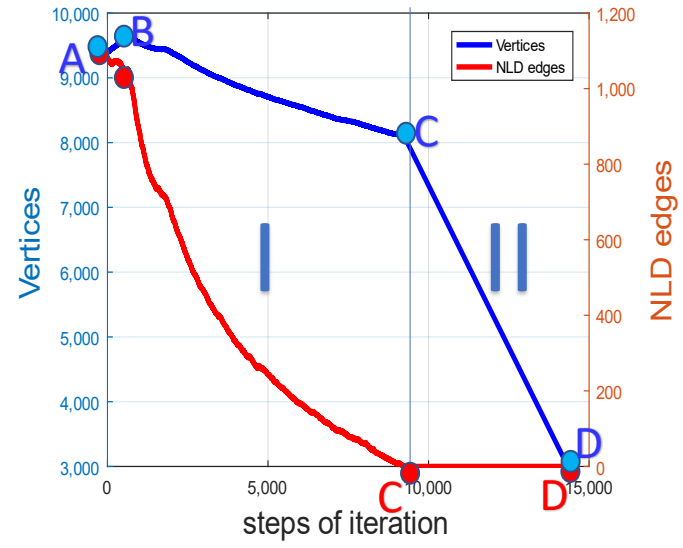

(a) Trend of vertices and NLD edges with steps of iteration

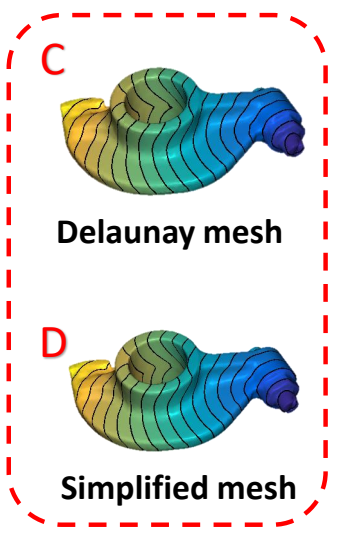

(c) Thermal diffusion simulation effect

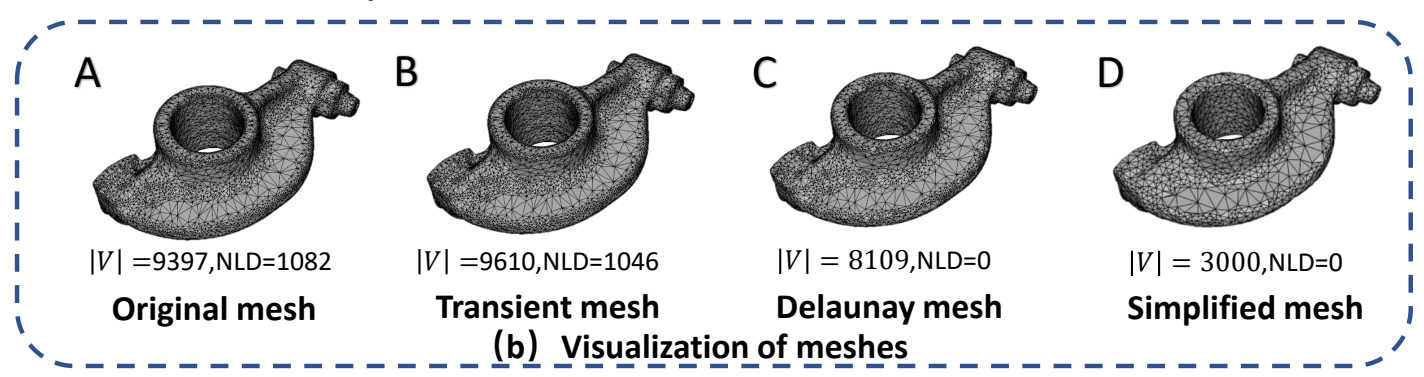

Figure 11. The results after the hybrid construction and simplification algorithm with the model of "rocker". 
The visualization of the meshes of A, B, C and D is shown in Figure 11b, respectively. As mentioned above, $A$ is the original mesh with 9397 vertices and 1082 NLD-edges, $B$ is a non-Delaunay meshes processed by the vertices inserting operation, $C$ is the Delaunay mesh obtained by the Algorithm 2 with 8109 vertices, and D is a simplified mesh with 3000 vertices. Especially, the thermal diffusion simulation results for $C$ and $D$ are also shown in Figure 11c, respectively. As the number of vertices reduce, the geometric features of the original mesh can still be effectively displayed. These results demonstrate the effectiveness of the simplification algorithm in preserving geometric information. In addition, the thermal diffusion rendering of the two simplified meshes also show that the constructed Delaunay meshes can also achieve a good performance in numerical simulation. For the case of $\mathrm{D}$, it is possible to obtain similar numerical simulation results in the case of $C$ with fewer vertices. It will enable the simplification of both the Delaunay mesh and the nonDelaunay mesh without destroying the performance of the thermal diffusion simulation.

In order to further illustrate the generality of Algorithm 2, three more experiments are conducted and shown in Figure 12. The processed models are "elephant", "horse", and "head" of non-Delaunay mesh, respectively. After the processing of Algorithm 2, all the meshes can be transformed into the Delaunay meshes and the number of vertices is reduced. To demonstrate the advantage of the simplification algorithm, the methods of the classical QEM [3] and the Delaunay mesh simplification [13] were conducted and illustrated as the contrast. All these two methods and the proposed method in this paper are simplified to the same number of vertices. The QEM method [3] is considered as a general control experiment for the simplification algorithm because of its good performance in the aspect of geometric feature preservation of the model. However, it is noteworthy that the results obtained with this method do not have the local and global properties of Delaunay mesh. There are still many narrow triangles among the simplified meshes, which are easy to be visually detected by its surface meshes. Although Liu's method [13] can preserve the local and global properties of the Delaunay meshes, it is visually noticeable that the meshes obtained by our method are more uniform with the same level of simplification.

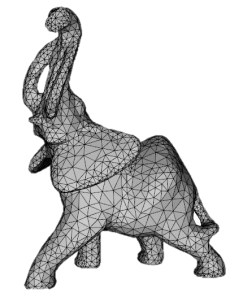

$|V|=2775, \mathrm{NLD}=16$

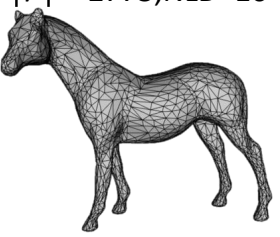

$|V|=2000, \mathrm{NLD}=823$

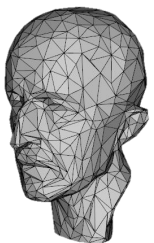

$|V|=664, \mathrm{NLD}=298$

(a) Original mesh

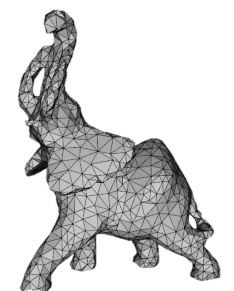

$|V|=1377, \mathrm{NLD}=0$

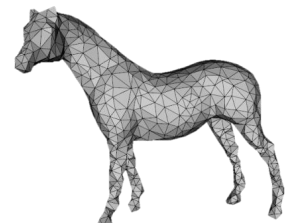

$|V|=878, \mathrm{NLD}=0$

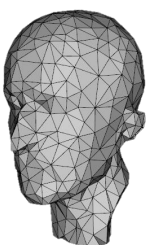

$|V|=516, \mathrm{NLD}=0$

(b) Our method
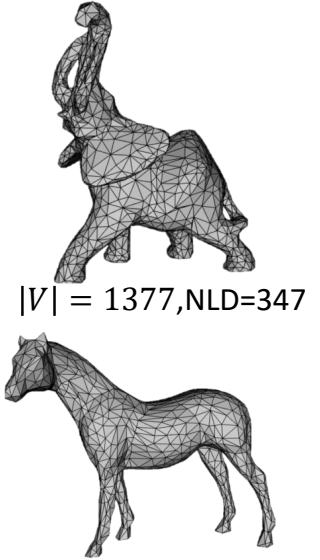

$|V|=878, \mathrm{NLD}=346$

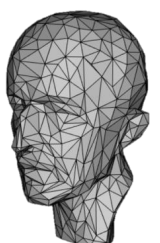

$|V|=516, \mathrm{NLD}=211$

(c) QEM method

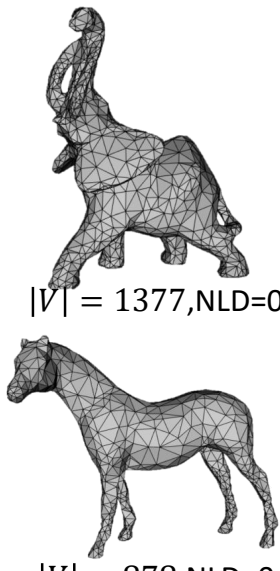

$|V|=878, \mathrm{NLD}=0$

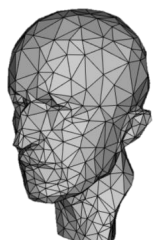

$|V|=516, \mathrm{NLD}=0$

(d) Liu's method

Figure 12. The comparison of the processed effects of the Delaunay mesh, (a) the original mesh, (b) our method, (c) QEM method, and (d) Liu's method. 
To better illustrate the preservation level of the geometric features after the simplification, the ratios surface-area-to-volume $r$ of these four models are calculated and listed in Table 1. The algorithm proposed in this paper is based on the principle of minimal volume destruction. It can be seen that the vertices of mesh gradually decreases when the quantity of the meshes is improved. As the illustration factor of geometric features, the quantification criterions $r$ of all these methods differ slightly. The values of $\Delta r$ are defined as the geometric feature variations of the meshes compared to the original mesh. The small $\Delta r$ value means that the changes to the geometric feature of the simplified mesh is little. The results show that the geometric features of all these meshes can be preserved after the simplification algorithm. In particular, for the original mesh with a large number of vertices, such as "rocker", "elephant", and "horse", the simplified mesh of the proposed method has smaller $\Delta r$ compared to the simplified meshes of other methods. However, since the proposed method is based on minimal volume destruction, when the original model of "head" with fewer vertices is simplified, the vertex collapsing may result in the undesired volume destruction. Therefore, the proposed method in this paper is not applicable to process meshes with few vertices. All in all, it is demonstrated that the method proposed in this paper can protect the local and global properties of the Delaunay mesh and preserve the geometric features of the original mesh in most cases.

Table 1. The geometric feature information of the meshes.

\begin{tabular}{cccccc}
\hline Meshes & Vertices & Volume & Surface Area & $\boldsymbol{r}$ & $\Delta \boldsymbol{r}$ \\
\hline rocker_original & 9397 & 0.0425 & 1.2965 & 30.4965 & 0 \\
rocker_our & 3000 & 0.0423 & 1.28926 & 30.4283 & 0.00223 \\
rocker_qem [3] & 3000 & 0.0424 & 1.30145 & 30.6283 & 0.00432 \\
rocker_liu [13] & 3000 & 0.0423 & 1.28805 & 30.4141 & 0.00270 \\
\hline elephant_original & 2775 & 0.046201 & 1.24496 & 26.9465 & 0 \\
elephant_our & 1377 & 0.045656 & 1.22927 & 26.925 & 0.00079 \\
elephant_qem [3] & 1377 & 0.04609 & 1.24725 & 27.061 & 0.00424 \\
elephant_liu [13] & 1377 & 0.045757 & 1.24068 & 27.11 & 0.00606 \\
\hline horse_original & 2000 & 0.157444 & 2.49807 & 15.8664 & 0 \\
horse_our & 878 & 0.153055 & 2.44337 & 15.964 & 0.00615 \\
horse_qem [3] & 878 & 0.156998 & 2.50834 & 15.9769 & 0.00696 \\
horse_liu [13] & 878 & 0.153371 & 2.46727 & 16.087 & 0.01390 \\
\hline head_original & 664 & 5.62153 & 0.183606 & 0.032661 & 0 \\
head_our & 516 & 5.60071 & 0.181661 & 0.032435 & $\mathbf{0 . 0 0 6 9 1}$ \\
head_qem [3] & 516 & 5.61976 & 0.183612 & 0.032673 & 0.00035 \\
head_liu [13] & 516 & 5.5796 & 0.18225 & 0.032664 & $\mathbf{0 . 0 0 0 0 7}$ \\
\hline
\end{tabular}

\section{Conclusions}

In this paper, the Delaunay construction and simplification algorithms were proposed to improve the model complexity and simulating accuracy. Firstly, we proposed two improved remeshing operations of the vertex inserting and the vertex collapsing. Then, the improved algorithms for the construction and simplification of Delaunay mesh based on minimal volume destruction were presented. The Delaunay construction algorithm can transform the arbitrary mesh into the Delaunay mesh by inserting finite numbers of vertices. The simplification algorithm can classify the vertices into collapsible and non-collapsible vertices, which will be performed differently according to the types of vertices. Moreover, the renderings of triangular meshes demonstrated the accuracy of the proposed algorithms. Finally, the experimental results showed that the algorithm can improve the quality of the arbitrary meshes. It is also noteworthy that, when the original model with fewer vertices is simplified, the vertex collapsing may result in the undesired volume destruction. The similar limitation occurs when the meshes with a large number of vertices were simplified to a high level, for example to $20 \%$ of the original vertices. Then, the cost of the algorithm 
will increase significantly and the geometric feature information of the simplified mesh may degenerate. To minimize the destruction to the original mesh, the control factor $\delta$ was designed to judge the narrow triangle. At present, the value of the control factor $\delta$ should be based on the user defined values. We will try to realize the automatic and real-time optimization of the control factor in the future study. In our future work, the proposed algorithms will be applied to 3D model processing for stereo-lithography additive manufacturing and experiments will be conducted to evaluate the performance.

Author Contributions: The paper's initial idea was proposed by Y.H., T.W., B.L. and G.W. The conceptualization is by Y.H. and T.W.; the software work and analysis were performed by Y.H., H.L., X.L. and Y.Z.; Supervision by T.W. and G.W. The original draft preparation was by Y.H. and T.W. and the review and editing were performed by Y.H. and T.W. All authors have read and agreed to the published version of the manuscript.

Funding: The authors gratefully acknowledge the financial support of the National Natural Science Foundation of China (Grant No. 52005479), the Technology and Engineering Center for Space Utilization, Chinese Academy of Sciences (Project No. CSU-JJKT-2020-6) and the Scientific Instrument Developing Project of the Chinese Academy of Sciences (Grant No. YJKYYQ20200068).

Conflicts of Interest: We declare that we have no financial and personal relationships with other people or organizations that can inappropriately influence our work, there is no professional or other personal interest of any nature or kind in any product, service and company that could be construed as influencing the position presented in, or the review of, the manuscript entitled.

\section{References}

1. Alliez, P.; Ucelli, G.; Gotsman, C.; Attene, M. Recent Advances in Remeshing of Surfaces. In Shape Analysis and Structuring; De Floriani, L., Spagnuolo, M., Eds.; Publishing House: Berlin/Heidelberg, Germany, 2008; pp. 53-82.

2. Hoope, H.; Derose, T.; Duchamp, T. Mesh optimization. In Proceedings of the Conference on Computer Graphics and Interactive Techniques, Anaheim, CA, USA, 2-6 August 1993.

3. Garland, M.; Heckbert, P. Surface Simplification Using Quadric Error Metrics. In Proceedings of the 24th Annual Conference on Computer Graphics and Interactive Techniques, Los Angeles, CA, USA, 3-8 August 1997.

4. Botsch, M.; Kobbelt, L. A Remeshing Approach to Multiresolution Modeling; Association for Computing Machinery: New York, NY, USA, 2004 ; pp. 189-196.

5. Wang, Y.Q.; Yan, D.M.; Liu, X.H.; Tang, C.C.; Guo, J.W.; Zhang, X.P.; Wonka, P. Isotropic Surface Remeshing without Large and Small Angles. IEEE Trans. Vis. Comput. Graph. 2019, 25, 2430-2442. [CrossRef] [PubMed]

6. Wang, Q.; Gao, B.; Wu, H. Triangular mesh generation on free-form surfaces based on bubble dynamics simulation. Eng. Comput. 2019, 36, 646-663. [CrossRef]

7. Khan, D.; Plopski, A.; Fujimoto, Y.; Kanbara, M.; Cheng, Z.; Kato, H. Valence optimization and angle improvement for molecular surface remeshing. Vis. Comput. 2020, 36, 2355-2368. [CrossRef]

8. Crane, K.; Weischedel, C.; Wardetzky, M. Geodesics in heat: A new approach to computing distance based on heat flow. ACM Trans. Graph. 2013, 32, 152-162. [CrossRef]

9. Bobenko, A.I.; Springborn, B.A. A Discrete Laplace-Beltrami Operator for Simplicial Surfaces. Discrete Comput. Geom. 2007, 38, 740-756. [CrossRef]

10. Ye, Z.; Yi, R.; Gong, W.; He, Y.; Liu, Y.-J. Dirichlet energy of Delaunay meshes and intrinsic Delaunay triangulations. Comput. Aided Des. 2020, 126, 102851. [CrossRef]

11. Dyer, R.; Zhang, H.; Möller, T. Voronoi-Delaunay Duality and Delaunay Meshes. In Proceedings of the 2007 ACM Symposium on Solid and Physical Modeling, Beijing, China, 4-6 June 2007.

12. Dyer, R.; Zhang, H.; Möller, T. Delaunay Mesh Construction. In Proceedings of the Fifth Eurographics Symposium on Geometry Processing, Barcelona, Spain, 4-6 July 2007.

13. Liu, Y.; Xu, C.; Fan, D.; He, Y. Efficient construction and simplification of Delaunay meshes. ACM Trans. Graph. 2015, 34, 174-186. [CrossRef]

14. Yi, R.; Liu, Y.; He, Y. Delaunay mesh simplification with differential evolution. ACM Trans. Graph. 2018, 37, 263-274. [CrossRef]

15. Rivin, I. Euclidean Structures on Simplicial Surfaces and Hyperbolic Volume. Ann. Math. 1994, 139, 553-580. [CrossRef]

16. Leibon, G; Letscher, D. Delaunay Triangulations and Voronoi Diagrams for Riemannian Manifolds. In Proceedings of the Annual Symposium on Computational Geometry, Hong Kong, China, 12-14 June 2000.

17. Chen, Z.; Wang, W.; Levy, B.; Liu, L.; Sun, F. Revisiting Optimal Delaunay Triangulation for 3D Graded Mesh Generation. SIAM J. Sci. Comput. 2014, 36, 930-945. [CrossRef]

18. Aurenhammer, F. Voronoi diagrams-A survey of a fundamental geometric data structure. ACM Comput. Surv. 1991, 23, 345-405. [CrossRef] 
19. Guibas, L.; Stolfi, J. Primitives for the manipulation of general subdivisions and the computation of Voronoi. ACM Trans. Graph. 1985, 4, 74-123. [CrossRef]

20. Liu, Y.; Fan, D.; Xu, C.; He, Y. Constructing Intrinsic Delaunay Triangulations from the Dual of Geodesic Voronoi Diagrams. ACM Trans. Graph. 2017, 36, 1-15. [CrossRef]

21. Liu, Y.; Wang, W.; Lévy, B.; Sun, F.; Yan, D.; Lu, L.; Yang, C. On centroidal voronoi tessellation-Energy smoothness and fast computation. ACM Trans. Graph. 2009, 28, 101-117. [CrossRef]

22. Wang, X.; Ying, X.; Liu, Y.; Xin, S.; Wang, W.; Gu, X.; Mueller-Wittig, W.; He, Y. Intrinsic computation of centroidal Voronoi tessellation (CVT) on meshes. Comput. Aided Des. 2015, 58, 51-61. [CrossRef]

23. Liu, Y.; Xu, C.; Yi, R.; Fan, D.; He, Y. Manifold differential evolution (MDE): A global optimization method for geodesic centroidal voronoi tessellations on meshes. ACM Trans. Graph. 2016, 35, 243-252. [CrossRef] 